\title{
Tratamento endovascular de pseudo-aneurisma da artéria subclávia em criança hemofílica
}

\author{
Endovascular treatment of subclavian artery pseudoaneurysm in a hemophiliac child
}

\author{
Emanuella G alvão de Sales e Silva', Ricardo Wagner da C osta M oreira², Elias Arcenio N eto², \\ Camila de Campos Silva ${ }^{3}$, Charles Edouard Zurstrassen ${ }^{4}$, Flávio Roberto Cavalleiro de M acedo Ribeiro ${ }^{4}$,

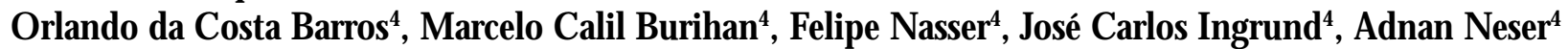

\begin{abstract}
Resumo
0 uso decateteres venosos centrais em pacienteshemofílicosémuito freqüente, devido às próprias características terapêuticas da doença. As complicações desses procedimentos, tais como pseudo-aneurisma, geralmente são mais graves nesses pacientes. A correção cirúrgica do pseudo-aneurisma que acomete a artéria subclávia constitui um dos maiores desafios da cirurgia vascular. Em pacientes hemofílicos, à dificuldade habitual de exposição cirúrgica somam-se os problemas de alteração no processo normal de coagulação. Como alternativa ao tratamento cirúrgico convencional, a utilização de técnicas endovasculares constitui uma solução segura e com bons resultados.
\end{abstract}

Palavras-chave: Falso aneurisma, angioplastia, hemofilia.

\section{Introdução}

A hemofilia A é uma doença hemorrágica grave decorrenteda deficiência defator VIII (FVIII) da coagulação, herdada através do gene localizado na porção 2.8 do cromossomo X. É classificada como grave quando os níveis plasmáticos do FVIII são menores que $1 \%$. Sua

1. Residente de cirurgia vascular, Hospital Santa Marcelina de Itaquera, São Paulo, SP.

2. Residente nível 3 de cirurgia vascular, Hospital Santa Marcelina de Itaquera, São Paulo, SP.

3. Hematologista, Hospital Santa Marcelina de Itaquera, São Paulo, SP.

4. Cirurgião vascular, Hospital Santa Marcelina de Itaquera, São Paulo, SP.

Trabalho apresentado sob a forma de pôster no $36^{\circ}$ Congresso Brasileiro de Angiologia e Cirurgia Vascular, realizado na cidade de Porto Alegre, no período de 4 a 8 de setembro de 2005 .

Artigo submetido em 20.02.06, aceito em 24.05.06.

\begin{abstract}
The placement of central venous catheters in hemophiliac patients is very frequent, dueto the specific treatment characteristics of the disease. The complications of these procedures, such as pseudoaneurysm, are generally worse in such patients. Surgical treatment of subclavian artery pseudoaneurysm is known to be one of the greatest surgical challenges in vascular surgery. In hemophiliac patients, the alterations in the normal cascade of coagulation are added to the difficult surgical exposure. As an alternative to the usual surgical treatment, the use of endovascular techniquesis a safe method and has good outcomes.
\end{abstract}

Key words: Aneurysm, false, angioplasty, hemophilia.

prevalência é de 1/20.000 indivíduos, quase que exclusivamente do sexo masculino, sendo rara sua apresentação em mulheres ${ }^{1,2}$.

Para pacientes com inibidores, isto é, que desenvolvem anticorpos da classe IgG contra o FVIII, estão disponíveis os concentrados de complexo protrombínico ecomplexo protrombínico parcialmenteativado, FVIII ativado recombinanteeprotocolos deimunotolerância, tratamentos imunossupressores que visam à erradicação desses anticorpos.

Em muitos casos, os pacientes hemofílicos vêemse diante de situações em que necessitam de infusões freqüentes de fator de coagulação, como aqueles que fazem imunotolerância, ou profilaxia de eventos hemorrágicos, segundo diversostrabalhosvigentes. M uitas vezes, são crianças que precisam receber o fator deficiente, em decorrência de hemorragia prévia do sistema nervoso central, por tempo prolongado, pre- 
venindo novos episódios, ou que estão recebendo altas doses de fator ou outros agentes para erradicar os anticorpos inibitórios da atividade coagulante. Para tanto, podem ser utilizados cateteres venosos na tentativa de garantir um acesso central rápido e fácil para as freqüentes reposições de fator. Eles permitem o início precoce da profilaxia eaté mesmo a terapia domiciliar ${ }^{3}$. Para ospacientesquetêm inibidores, principalmenteos dealto título, oscateteresestão bem indicados, visto que com maior freqüência precisam da reposição defator ou estão incluídos nos estudos de imunotolerância ${ }^{3}$.

O scateteres podem ser totalmenteimplantáveis no tecido subcutâneo, como o port-a-cath, ou semi-implantáveis, como o de H ickman 4 .

A colocação do cateter em pacientes hemofílicos geral mente é bem-sucedida mediante a adoção de protocolos de profilaxia. D essa forma, é possível realizar um procedimento seguro, minimizando o risco de formação de hematomas adjacentes ao reservatório de um cateter (evitando também eventosinfecciosos) eaté mesmo hemorragias graves.

N o entanto, apesar de ser um método efetivo, que garante eficiência e flexibilidade na sua utilização, há o risco das complicações inerentes a ele, tais como infecções e trombose ${ }^{5}$. Essa obstrução pode ocorrer devido ao depósito intraluminal de fibrina ou outras substâncias que precipitam ${ }^{3}$.

A punção para obtenção de uma veia central geralmente é realizada na veia subclávia ou jugular interna. T al procedimento não éisento deriscos. Complicações, taiscomo pneumotórax, hemotórax edesenvolvimento de pseudo-aneurisma após punção arterial inadvertida, podem ocorrer. Segundo Casado-Flores, em crianças internadas em uma unidade de terapia intensiva, a freqüência de complicações relacionadas ao implante de cateteres é de $22 \%$, sendo 2,9\% graves (pneumotórax- $1,9 \%$; hemotórax $-1 \%)^{6}$. 0 acesso daveiajugular interna está associado a um menor índice de mau posicionamento do cateter e maior incidência de punção arterial em relação à punção da veia subclávia7.

Em situações de emergência em que 0 acesso venoso periférico não é possível, a utilização de cateteres centrais de curta permanência se faz necessária. $\mathrm{N}$ esses casos, a profilaxia é mandatória. $\mathrm{No}$ entanto, quando não há disponibilidade dos fatores de coagulação ou quando não há conhecimento do diagnóstico, os riscos para o paciente são maiores, podendo levar até ao óbito.
N o presente artigo, os autores relatam um tratamento por técnica endovascular em criança hemofílica A grave com inibidor.

\section{Relato de caso}

Menor de 1 ano de idade, do sexo masculino, encaminhado de outro serviço em pós-operatório de craniotomia descompressiva para drenagem de hematoma decorrente de trauma, com diagnóstico associado de hemofilia A grave. Apresentava ainda grande pseudo-aneurisma em região supraclavicular direita decorrente da tentativa de acesso venoso (Figura 1). 0 pseudo-aneurisma, devido à compressão da traquéia, causava importante desconforto respiratório.

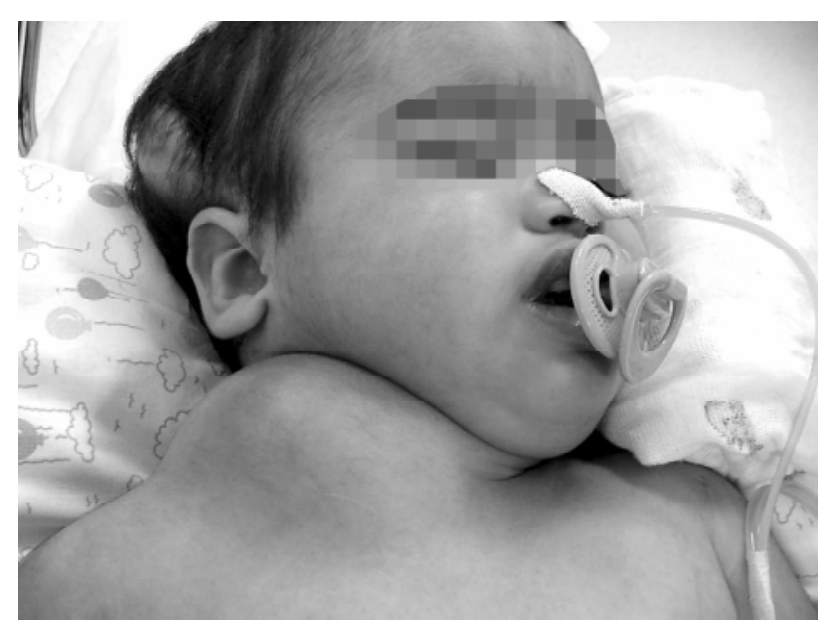

Figura 1 - G Gande massa pulsátil em região supraclavicular direita

0 estudo por angiorressonância identificou grande pseudo-aneurisma originário da artéria subclávia direita, próximo à origem da artéria vertebral (Figura 2). Dada a precária condição clínica do paciente, optou-se pelo tratamento endovascular percutâneo por acesso da artéria femoral comum direita, utilizando-se infusão prévia de fator de coagulação em dose profilática (100 U/kg) e com manutenção da mesma dose por via intravenosa de 12 em 12 horas.

Realizou-se punção da artéria femoral comum direita com colocação deintrodutor $4 \mathrm{~F}$ pediátrico, seguido de estudo angiográfico do tronco supra-aórtico, confirmando os achados da angiorressonância. Através 


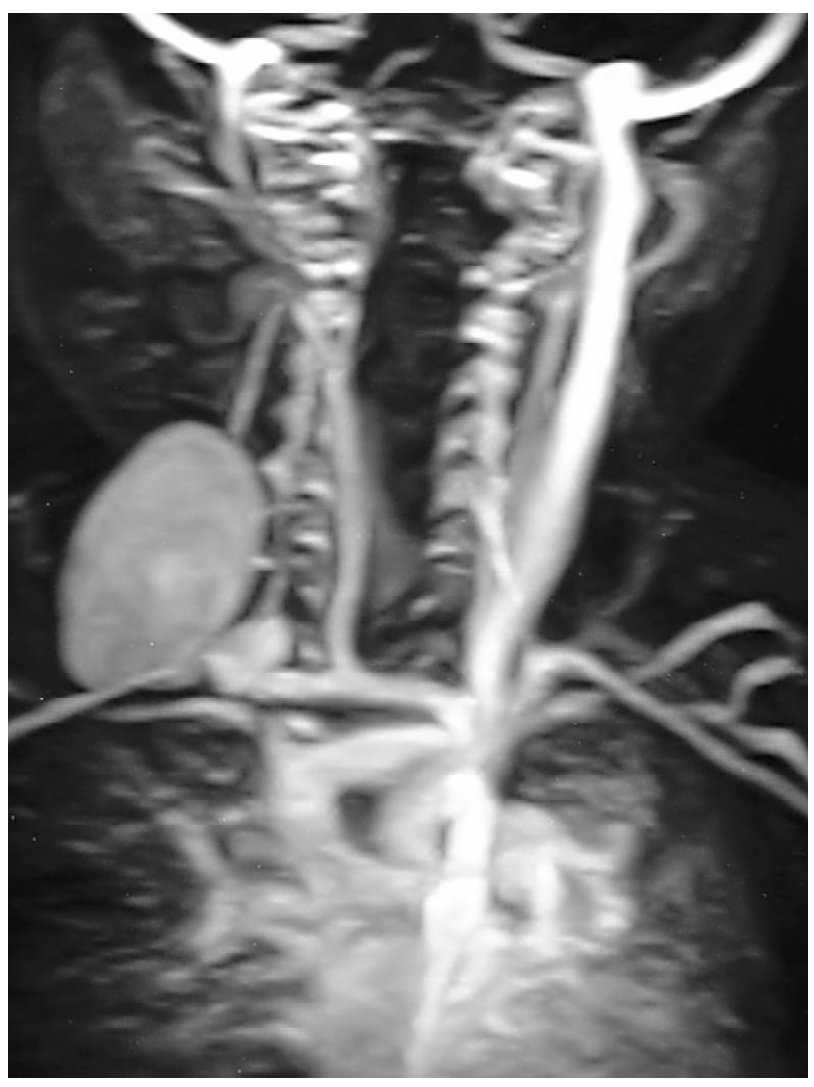

Figura 2 - Aspecto do pseudo-aneurisma em exame de angiorressonância

deste acesso, foi introduzido cateter-guia, posicionado no nível do tronco braquiocefálico. A lesão foi transposta com fio-guia hidrofílico 0.014 " sob roadmapping, seguida de implante primário de endoprótese J ostent 3 x $19 \mathrm{~mm}$.

A arteriografia de controle evidenciou adequado posicionamento da endoprótese e tratamento da lesão com preservação da artéria vertebral (Figura 3). I mediatamente após o procedimento, observou-se ausência de pulsatilidade na região tumoral.

A criança evoluiu no pós-operatório imediato he modinamicamente estável, com presença de pequeno sangramento no local de punção da artéria femoral direita, tratado de maneira conservadora com compressão local.

$\mathrm{N}$ os dias subseqüentes, o menor continuou rece bendo reposição de fator de coagulação na tentativa de correção dos parâmetros laboratoriais do coagulograma, utilizando-se uma dose total de $41.000 \mathrm{U}$, acarretando um custo total de aproximadamente US\$ $65.000,00$. N 0 entanto, não foi observada redução adequada dos valores de tempo de tromboplastina parcial ativada (TTPA), evidenciando a atividade do anticorpo inibidor. Paralelamente, evoluiu para agravamento do trauma cranioencefálico, com desenvolvimento de hidrocefalia.

No sexto dia de pós-operatório, devido à evolução insatisfatória do quadro respiratório e manutenção do desvio da traquéia, optou-se pela drenagem do hematoma cervical. Durante o procedimento cirúrgico, foi retirado gran dehematoma encapsulado, observando-se sangramento difuso em toda a área cruenta. Evoluiu, então, com sangramento da região femoral direita e da incisão cirúrgica supraclavicular direita, além de apresentar parâmetros laboratoriais compatíveis com coagulação intravascular disseminada (CIVD ). H ouve piora do quadro neurológico, evoluindo para o coma.
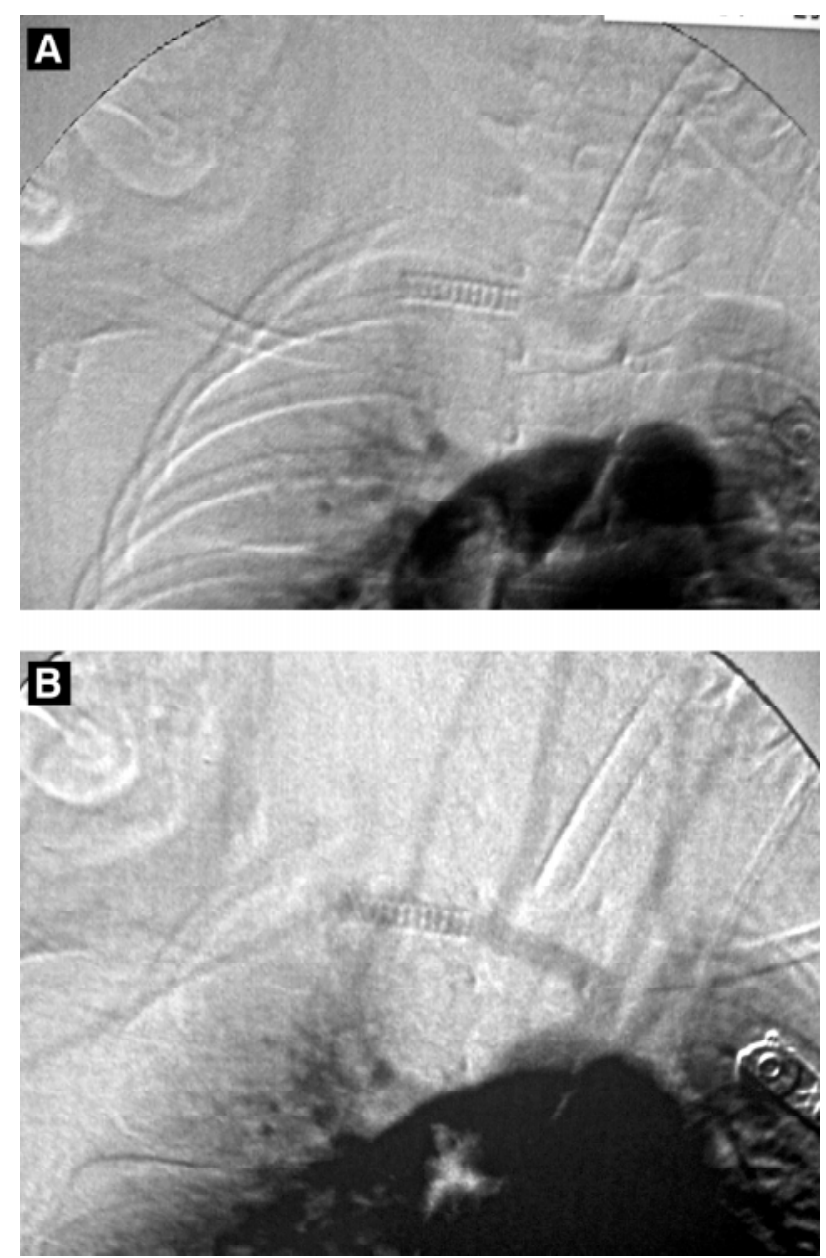

Figura 3 - Aspecto final após correção do pseudo-aneurisma com uso de stent revestido 
M esmo após infusão de fatores da coagulação, na tentativa de corrigir o sangramento, houve piora progressiva, e a criança evoluiu para o óbito no $12^{\circ}$ dia de implante da endoprótese.

\section{D iscussão}

A cateterização venosa central consistena colocação de um cateter na veia cava superior ou inferior através deuma veiacalibrosaqueconflui paraum dessesvasos ${ }^{8}$. A principal finalidade da inserção central de um cateter é para a administração de medicamentos, hemoderivados e verificação de pressão venosa central. Em pacientes hemofílicos, principalmente crianças, o uso de um cateter central, especialmente os que são totalmente implantáveis, facilitam a administração freqüente de fatores da coagulação. Em pacientes hemofílicos com inibidores, que necessitam de tratamento para sangramentosespontâneoseterapia deimunotolerância, o uso de dispositivostotalmenteimplantáveis torna-seo procedimento ideal ${ }^{9}$.

Algumas recomendações sobre a utilização de dispositivos de acesso venoso central (DAVC) em pacientes hemofílicos podem ser retiradas de consenso publicado recentemente por Ewestein na revista $\mathrm{H}$ aemophilia9

Sobre a sel eção adequada dos pacientes, os prestadores de serviço de saúde devem dar preferência ao acesso venoso periférico sempre que possível, e a presença de uma infecção ativa, incluindo cáries, constitui uma contra-indicação absoluta ao implante dos DAVC.

Em indivíduos sem inibidores, o port-a-cath é preferível ao dispositivo externo. Um registro detalhado deve ser mantido, incluindo tipo de DAVC, nome e número de série do D AVC, data do implante, responsável pelo implante, localização anatômica, tipo de regime terapêutico, tipo e evolução das complicações, data e motivo da retirada 9 .

O s cuidados no manuseio dos D AVC também são de suprema importância. Todos os dispositivos devem receber uma infusão de solução salina ou heparina após cada uso, mantendo pressão positiva após cada infusão. T anto iodopovidona quanto clorexidina podem ser usadas como anti-sépticos tópicos para a pele; porém, existem evidências quemostram queo uso declorexidina pode estar associado a um menor número de infecções relacionadas ao cateter, especialmente em pacientes com história de infecção $0^{9}$.
A lavagem das mãos e a utilização constante de técnicas assépticas são os principais itens na prevenção de infecção relacionadas ao cateter. Os pacientes com D AVC necessitam de antibióticos profiláticos quando são submetidos a procedimentos invasivos, tais como cirurgias e extrações dentárias. T ambém é importante lembrar queum dispositivo não deveser rotineiramente trocado apenas com o propósito de reduzir o risco de infecção. 0 tratamento precoce eagressivo deinfecções é importante, independente do local.

A remoção de um DAVC devido a infecção é recomendada nas seguintes situações: persistência de hemoculturas positivas após uso apropriado de antibióticos sistêmicos; infecções causadas por cândida; infecções recorrentes causadas pelo mesmo organismo, apesar do uso de antibióticos adequados; infecção do túnel e da loja; erosão da pele sobre o port-a-cath; e se a bacteremia não se resolve após tratamento com antibióticos por 48 a 72 horas 9 .

0 utro item muito importante é a presença de trombose venosa. Todos os profissionais que lidam com pacientes em uso de um DAVC devem sempre manter um alto índice de suspeita clínica em relação à presença de uma trombose relacionada ao cateter. A pesquisa de rotina de trombofilia antes do implante de um D AVC não énecessária, a não ser que haja história de trombose venosa não relacionada ao cateter. 0 D AVC usado deve ter o cateter com o menor diâmetro possível necessário para uma terapia adequada, e a ponta do cateter deveficar posicionada no terço inferior da veia cava superior.

Q uando há suspeita de trombose venosa, uma venografia deve ser realizada quando exames de imagem não-invasi vos não conseguem confirmar o diagnóstico. Se o paciente ainda tem necessidade de uso do D AV C e a trombose é reduzida ou pouco sintomática, o cateter pode ser mantido. Se a retirada do DAVC não comprometer o tratamento, o mesmo deve ser retirado ${ }^{9}$.

Pseudo-aneurismas de grandes vasos e da artéria subclávia são raros após punção central, podendo ocorrer em 0,05 a 2\% dos casos. Suas complicações incluem expansão e compressão de estruturas respiratórias e neurovasculares adjacentes, rotura, trombose e erosão para pele com sangramento externo ${ }^{10,11}$.

Este paciente era portador de um grande pseudoaneurisma, quese encontrava em franca expansão, uma vez que possuía um distúrbio de coagulação grave, a 
hemofilia tipo A com inibidor, o que não permitiu a trombose espontânea do mesmo. Provavelmente, havia compressão e desvio da traquéia pelo pseudo-aneurisma, que seria a causa do desconforto respiratório inicial do paciente.

0 tratamento dospseudo-aneurismas deartérias de grande calibrepode ser cirúrgico ou tratado por técnica endovascular. A pesar de al guns autores acreditarem ser o tratamento cirúrgico o procedimento deescolha, uma vez que apresentam bons resultados, o reparo endovascular vem sendo relatado na literatura e tem sido mais utilizado na última década10-12.

No tratamento cirúrgico do pseudo-aneurisma da artéria subclávia, o controle proximal pode necessitar da exposição da artéria subclávia retroclavicular, com ou sem toracotomia, havendo risco de lesão deestruturas nobres como o nervo frênico ou ducto torácico (à esquerda) e o plexo braquial, além de hemorragia durante a dissecção ${ }^{11}$.

Particularmente neste caso, o reparo endovascular foi a mel hor opção, uma vez queo controlecirúrgico da lesão seriadifícil, não somentepel o tamanho da criança, na qual o espaço supraclavicular encontrava-seocupado pelo pseudo-aneurisma, como também pelo risco de sangramento incontrolável durante 0 ato cirúrgico, decorrente da hemofilia com inibidor da qual era portadora.

$\mathrm{N}$ ão encontramos, na literatura, relatos da utilização de stents em crianças para tratamento de pseudoaneurismas arteriais periféricos.

$M$ ais recentemente, al guns autores relataram o uso de injeção de trombina guiada por ultra-som como tratamento de pseudo-aneurisma e utilizaram essa técnica em três crianças, sendo duas com pseudo-aneurisma de artéria femoral e uma com pseudo-aneurisma de artéria epigástrica ${ }^{13}$.

Lidell relatou com sucesso o tratamento depseudoaneurisma de artéria pulmonar bilateral em criança de 5 anos com embolização ${ }^{14}$.

H á várias publicações de uso do tratamento endovascular com stents para doenças cardíacas congênitas, como estenoses de artéria pulmonar, coarctação da aorta, shunts aórtico-pulmonares e ducto arterial. São relatadas complicações como fratura do stent, migração, formação de aneurismas e reestenose intra-stent, porém são raras, e a maioria dos autores concorda que o uso do stent para tratamento dessas doenças é seguro e eficaz em pacientes selecionados ${ }^{15-17}$.
M errot et al. relataram a utilização de angioplastia com stent em duas crianças com dissecção de artéria renal pós-trauma, o que constituiu uma alternativa à cirurgia naquele momento, com sucesso imediato ${ }^{18}$.

Já em relação ao sangramento decorrido do processo cirúrgico, novo produto poderia ter sido utilizado na tentativa de cessação do sangramento, se estivesse disponível, como é o caso do N ovoSeven ${ }^{\circledR}$, fator VII de coagulação humana ativado recombinante (rFVIIa), desenvolvido para o tratamento de sangramento espontâneo e cirúrgico de pacientes hemofílicos congênitos portadores de anticorpos contra os fatores de coagulação VIII e IX e pacientes portadores de hemofilia adquirida.

O N ovoSeven induz a hemostasia local através da formação de complexos com o fator tissular e pela ativação direta do fator $X$ sobre a superfície das plaquetas ativadas. D esta forma, obtém-se a geração de trombina, levando à formação de um coágulo estável. V ários estudos realizados relatam a utilização desse produto em hemorragias do sistema nervoso central, situação muito grave em pacientes hemofílicos, principalmente se forem portadores de inibidores. Foi também demonstrado que o rFVII a promoveuma rápida eeficiente correção da coagulação em pacientes com lesão hemorrágica encefálica. É utilizado tanto nos episódios hemorrágicos agudos quanto na prevenção de manifestações hemorrágicas em cirurgias el etivas.

A hemorragia intracerebral está associada a alta mortalidade, eo tempo decorrido entrea sua instalação e 0 início de tratamento tem grande influência na capacidade do produto rFVII a em limitar a extensão da hemorragia. 0 s mel hores resultados encontram-se nos pacientes cuja intervenção se deu em até 3 horas do início dos sintomas. M ayer et al. ${ }^{19}$ demonstraram a redução da mortalidade, bem como de seqüelas neurológicas, em um grupo de pacientes não-hemofílicos. Em uma população hemofílica, Arkin $^{20}$ verificou que rFVII a controlou a hemorragia intracraniana de forma efetiva, edemodo semelhante, Rice\& Savidge ${ }^{21}$ observaram eficácia em $84 \%$ dos seus pacientes, portadores de coagulopatia hereditária e com sangramento em sistema nervoso central.

$\mathrm{N}$ o caso relatado, o tratamento endovascular com stent revestido foi o mais factível, em função dos riscos já explicitados anteriormente, e mostrou-se seguro e eficaz para o tratamento depseudo-aneurisma na criança. $\mathrm{N}$ ão sabemos ao certo qual seria o comportamento de um stent implantado em um vaso periférico que irá 
sofrer os efeitos do crescimento corporal, pois os relatos existentes na literatura são de correção de pseudoaneurisma em adolescentes e adultos.

\section{Referências}

1. Brettler DB, Levine PH . C linical manifestations and therapy of inherited coagulation factors deficiencies. In: Colman RW, $H$ irsh J, M arder V, Salzman EW, editors. Hemostasis and thrombosis: basic principles and clinical practice. 3rd ed. Philadelphia: JB Lippincott; 1994. p. 169-83.

2. Rodgers $G M, G$ reenberg $C S$. Inherited coagulation disorders. In: Lee GR, Foerster J, Lukens J, Paraskevas F, Greer JP, Rodgers GM , editors. Wintrobe's Clinical $\mathrm{H}$ ematology 10 th ed. Baltimore: W illiams \& Wilkins; 1999. p. 1682-732.

3. D omm JA, H udson M G, Janco RL. Complications of central venous access devices in paediatric haemophilia patients. H aemophilia. 2003;9:50-6.

4. M orado M, Jimenez-YusteV, Villar A, et al. Complications of central venous catheters in patients with haemophilia and inhibitors. H aemophilia. 2001;7:551-6.

5. Tarantino M D, Lail A, D onfield SM, et al. Surveillance of infectious complications associated with central venous access devices in children with haemophilia. $\mathrm{H}$ aemophilia. 2003;9:588-92.

6. Casado-Flores J, BarjaJ, M artino R, Serrano A, V aldivielso A. Complications of central venous catheterization in critically in children. Pediatr Crit Care M ed. 2001;2:57-62.

7. Ruesch $S, W$ alder $B, T$ ramer M R. Complications of central venous catheters: internal jugular versus subclavian access - a systematic review. Crit C are M ed. 2002;30:454-60.

8. Lassance $P, N$ eto $A S, N$ éri $E M$. C ateterizações em pediatria. In: Phol FF, Petroianu A. Tubos, sondas e drenos. Rio de J aneiro: G uanabara K oogan; 2000. p. 57-65.

9. Ewestein BM, Valentino LA, J ourneycakeJ $M$, et al. Consensus recommendations for use of central venous access devices haemophilia. H aemophilia. 2004;10:629-48.

10. Peces R, N avascues RA, Baltar J, Laures AS, Alvarez-Grande J. Pseudoaneurysm of the thyrocervical complicating percutaneous internal jugular-vein catheterization for hemodialysis. N ephrol Dial Transplant. 1998;13:1009-11.

11. Akgun S, Civelek A, Baltacioglu F, Ekici G. Successful endovascular repair of a subclavian artery pseudoaneurysm. N ephrol Dial Transplant. 1999;14:2219-21.
12. Criado $E, M$ artson WA, Ligush J, M auro M A, Keagy BA. Endovascular repair of peripheral aneurysms, pseudoaneurysms, and arteriovenous fistulas. Ann V asc Surg. 1997;11:253-63.

13. Pelchovitz DJ, Cahill AM, Baskin KM, Kaye RD, Towbin RB. Pseudoaneurysm in children: diagnosisand interventional management. Pediatr Radiol. 2005;35:434-9.

14. Liddell RP, Patel S, Neuwirth M, H ebert J, Arepally A, $\mathrm{H}$ ofmann LV. Transcatheter coil embolization of large pulmonary artery pseudoaneurysm in a child. J V asc Interv Radiol. 2003;14:923-7.

15. Celiker A, Bilgic A, Karagoz T, Pac A. Endovascular stent implantation in congenital heart defects. Turk J Pediatr. 2001;43:59-64.

16. Pass RH, H su DT, G arabedian CP, Schiller M S, Jayakumar $K A, H$ ellenbrand W E. Endovascular stent implantation in the pulmonary arteries of infants and children without the use of a long vascular sheath. Catheter C ardiovasc Interv. 2002;55: 505-9.

17. O kubo M, Benson LN . Intravascular and intracardiac stents used in congenital heart disease. Curr O pin C ardiol. 2001;16: 84-91.

18. M errot $T$, Portier $F, G$ alinier $P$, et al. Trauma of the renal pedicle in children. Report of 2 cases of late revascularization with endovascular prosthesis. Prog U rol. 2000;10:277-81.

19. M ayer SA, B run N C, Begtrup K, et al. Recombinant activated factor VII for acute intracerebral hemorrhage. N Engl J M ed. 2005;352:777-85.

20. Arkin $\mathrm{S}, \mathrm{C}$ ooper H A, H utter JJ, et al. Activated recombinant human coagulation factor VII therapy for intracranial hemorrhagein patientswith hemophiliaA or B with inhibitors. Results of the NovoSeven emergency-use program. H aemostasis. 1998;28:93-8.

21. RiceK M, Savidge G F. N ovoSeven (recombinant factor VIIa) in centeral nervous systems bleeds. H aemostasis. 1996;26 Suppl 1:131-4.

\section{Correspondência:}

Felipe $\mathrm{N}$ asser

Alameda Barão de Limeira, 1362/42 - Campos Elíseos

CEP 01202-002 - São Paulo, SP

Tel.: (11) 8224.0505

E-mail: nasser.felipe@gmail.com 